\title{
Soluciones solitónicas e instantónicas en teorías de Yang-Mills
}

\author{
H. A. Benítez, M. Culqui, R. Negrón y F. Villegas* \\ Facultad de Ciencias Físicas, Universidad Nacional Mayor de San Marcos, Ap-Postal 14-0149, Lima 14, \\ Perú.
}

Recibido 3 marzo 2011 - Aceptado 29 junio 2011

\begin{abstract}
Presentamos la teoría de gauge no abeliana en el grupo $\mathrm{SU}(2)$ y estudiamos soluciones tipo solitón e instantón en teorías de campos y su correspondencia. Las soluciones clásicas en el espacio euclidiano y de Minkowski para campos de Yang-Mills son analizadas obteniendo instantones y monopolos, respectivamente.
\end{abstract}

Palabras claves: Yang-Mills, Solitón, Instantones, Monopolos..

\section{Soliton and Instanton Solutions in Yang-Mills Theories}

We introduce non-abelian gauge theory in $\mathrm{SU}(2)$ group and we study the instanton and the soliton as solutions in the field theory and their correspondence. In these Yang-Mills solutions in the Minkowski and euclidean space we find instantons and monopole behaviour.

Keywords: Yang-Mills, soliton, instanton, monopoles..

\section{Introducción}

El modelo propuesto por Yang y Millls en 1954 [1] para el estudio de las interacciones con invariancia local, teorías gauge, obtuvo gran suceso convirtiéndose la base fundamental en el estudio de las interacciones en la física de partículas al poder explicar elegantemente la interacción electromagnética, y luego bajo el mecanismo de Higgs, la unificación de la interacción electro-débil basado en el grupo $\mathrm{S}(2) \times \mathrm{U}(1)$, modelo de WeinbergSalam, así como la cromodinámica cuántica basada en una teoría de Yang-Mills para el grupo SU(3) para el confinamiento de los quarks.

Los solitones e instantones aparecen como soluciones de la ecuación de campo en teorías de Yang-Mills. Los solitones aparecen como soluciones en el espacio de Minkowki para configuraciones con energía finita. Un caso particular de soluciones solitónicas es el de una teoría gauge no abeliana acoplada a un campo de Higgs, modelo de Georgi Glashow, de donde se desprenden las soluciones del tipo monopolo magnético $[2,3]$.

Las soluciones instantónicas son obtenidas en configuraciones del espacio euclideano con la restricción de una acción finita. Los instantones se encuentran relacionados con el proceso de tunelaje es el espacio de Minkowski.

\footnotetext{
*fvillegass@gmail.com
}

\section{Teorías de gauge no Abelianas}

Considerando un campo fermiónico doblete de spin $1 / 2$ como

$$
\Psi=\left(\begin{array}{c}
\psi_{1} \\
\psi_{2}
\end{array}\right)
$$

El lagrangiano para este modelo, escrito de forma compacta se describe como

$$
\begin{aligned}
\mathcal{L} & =\bar{\psi}_{1}\left(i \gamma^{\mu} \partial_{\mu}-m_{1}\right) \psi_{1}+\bar{\psi}_{2}\left(i \gamma^{\mu} \partial_{\mu}-m_{2}\right) \psi_{2} \\
& =\bar{\Psi}\left(i \gamma^{\mu} \partial_{\mu}-m\right) \Psi,
\end{aligned}
$$

donde $m=\operatorname{diag}\left(m_{1}, m_{2}\right)$ es la matriz de masas. Es necesario notar que este lagrangiano posee invariancia ante las transformaciones de gauge globales como

$$
\Psi(x) \rightarrow U(\alpha) \Psi(x),
$$

considerando para nuestro caso, $U(\alpha)=e^{i \tau^{a} \alpha_{a}}$ como elementos del grupo SU(2), y $\tau^{a}$ como las matrices de Pauli que satisfacen el algebra

$$
\left[\frac{\tau^{a}}{2}, \frac{\tau^{b}}{2}\right]=i \epsilon_{a b c} \frac{\tau^{c}}{2} .
$$

El término con la derivada, Ec.(1), posee ahora la transformación

$$
\bar{\Psi} \gamma^{\mu} \partial_{\mu} \Psi \rightarrow \bar{\Psi} \gamma^{\mu} \partial_{\mu} \Psi-i \bar{\Psi} \gamma^{\mu} \partial_{\mu} \alpha(x) \Psi
$$


El segundo término quiebra la invariancia del lagrangiano bajo la transformación y requiere que posea una invariancia local.

Definiendo la derivada covariante como

$$
D_{\mu}=\partial_{\mu}-i g \frac{\tau \cdot A_{\mu}}{2}
$$

que posee la transformación

$$
D_{\mu} \Psi \rightarrow U(\alpha) D_{\mu} \Psi,
$$

obtenemos

$$
\left(\partial_{\mu}-i g \frac{\tau \cdot A_{\mu}^{\prime}}{2}\right)(U(\alpha) \Psi)=U(\alpha)\left(\partial_{\mu}-i g \frac{\tau \cdot A_{\mu}}{2}\right) \Psi,
$$

de modo que

$$
\frac{\tau \cdot A_{\mu}^{\prime}}{2}=U(\alpha) \frac{\tau \cdot A_{\mu}}{2} U^{-1}(\alpha)-\frac{1}{g}\left[\partial_{\mu} U(\alpha)\right] U^{-1}(\alpha),
$$

definimos $A^{\mu}=\tau^{a} A_{a}^{\mu}$.

Luego, el lagrangiano invariante ante las transformaciones de gauge locales tiene la forma

$$
\mathcal{L}=\bar{\Psi}\left(i \gamma^{\mu} D_{\mu}-m\right) \Psi-\frac{1}{2} \operatorname{Tr}\left(F_{\mu \nu} F^{\mu \nu}\right),
$$

donde definimos el tensor de campo de gauge $F_{\mu \nu}$ como

$$
F_{\mu \nu}=\partial_{\mu} A_{\nu}-\partial_{\nu} A_{\mu}+i g\left[A_{\mu}, A_{\nu},\right],
$$

que también posee una invariancia de gauge local de

$$
F_{\mu \nu} \rightarrow U(\alpha) F_{\mu \nu} U^{-1}(\alpha) .
$$

El dual del tensor del campo $\widetilde{F}^{\mu \nu}$ es definido como

$$
\widetilde{F}^{\mu \nu}=\frac{1}{2} \epsilon_{\mu \nu}^{\rho \sigma} F_{\rho \sigma},
$$

debido a la identidad de Bianchi expresado por

$$
D_{\mu} \widetilde{F}^{\mu \nu}=0 .
$$

\section{Soluciones solitónicas en el modelo de Georgi-Glashow}

\section{Solitones}

Las soluciones localizadas son aquellas soluciones de las ecuaciones de campo cuya densidad de energía $\epsilon(x, t)$, en cualquier tiempo finito $t$, son finitas en algunas regiones finitas del espacio y se aproxima a cero en el infinito, lo suficientemente rápido, como para que sea integrable. De esta manera definimos una onda solitaria como aquella solución localizada no singular de una ecuación de campo no lineal cuya densidad de energía se ecuentra localizada y posee una dependencia espaciotemporal de la forma

$$
\epsilon(\boldsymbol{x}, t)=\epsilon(\boldsymbol{x}-\boldsymbol{u}, t),
$$

donde $\boldsymbol{u}$ es la velocidad.

La ecuación anterior define una onda solitaria en una o mas dimensiones espaciales. Para una solución localizada e independiente del tiempo su velocidad debe ser cero, $\boldsymbol{u}=\mathbf{0}$.

Considerando una solución tipo onda solitaria para la ecuación no lineal cuya densidad de energía es $\epsilon_{0}(\boldsymbol{x}-\boldsymbol{u}, t)$, la evolución del sistema cuando $t \rightarrow-\infty$ permite $N$ ondas solitarias de posiciones y velocidades arbitrarias. La densidad de energía de estas soluciones tiene la forma

$$
\epsilon(\boldsymbol{x}, t) \rightarrow \sum_{i=1}^{N} \epsilon_{0}\left(\boldsymbol{x}_{i}-\boldsymbol{a}_{i}-\boldsymbol{u}_{i}, t\right) \quad t \rightarrow-\infty
$$

Con esta configuración, la evolución en el tiempo está dada por la ecuación del campo. Un solitón es una onda solitaria cuyos perfiles de energía son asintóticamente devueltos a sus formas y velocidades originales.

\section{Modelo de Georgi-Glashow}

Este modelo se analiza mediante la solucion encontrada independientemente por 't Hooft [2,3] en 1974. Bajo una teoría de Yang-Mills en el grupo SU(2), acoplada a campos escalares en la representación adjunta obtenemos soluciones que presentan solitones como soluciones a las ecuaciones de movimiento. También, este modelo presenta monopolos magnéticos como soluciones.

El modelo consiste de un triplete de campos escalares de Higgs $\phi^{a}(x, t)$ y campos de gauge $A_{\mu}^{a}(x, t)$ en $(3+1)$ dimensiones. La densidad lagragiana invariante ante transformaciones de gauge $\mathrm{SU}(2)$ locales para el sector bosónico está dado por

$$
\mathcal{L}=-\frac{1}{2} \operatorname{Tr}\left(F_{\mu \nu} F^{\mu \nu}\right)+\operatorname{Tr}\left(D_{\mu} \phi D^{\mu} \psi\right)-\frac{1}{4} \lambda\left(\phi^{a} \phi^{a}-a^{2}\right)^{2}=-\frac{1}{4} F_{\mu \nu}^{a} F^{a \mu \nu}+\frac{1}{2}\left(D_{\mu} \phi^{a}\right)\left(D^{\mu} \phi^{a}\right)-\frac{1}{4} \lambda\left(\phi^{a} \phi^{a}-a^{2}\right)^{2} .
$$


Aquí, $F_{\mu \nu}=F_{\mu \nu}^{a} \tau^{a}, \phi=\phi^{a} \tau^{a}$, donde $\tau^{a}$ es el generador del grupo $\mathrm{SU}(2)$, que están expresados en la representación adjunta como $\left(\tau^{a}\right)_{a b c}=-i \epsilon_{a b c}$.

El tensor de campo de gauge expresado como

$$
F_{\mu \nu}^{a}=\partial_{\mu} A_{\mu}^{a}-\partial_{\nu} A_{\nu}^{a}+g \epsilon_{a b c} A_{\mu}^{b} A_{\nu}^{c},
$$

que en forma matricial se representa mediante

$$
F_{\mu \nu}=\partial_{\mu} A_{\nu}-\partial_{\nu} A_{\mu}+i g\left[A_{\mu}, A_{\nu}\right]=\frac{1}{i e}\left[D_{\mu}, D_{\nu}\right] .
$$

La derivada covariante $D_{\mu} \phi^{a}$, donde el operador se define como

$$
D_{\mu}=\partial_{\mu}+i g A_{\mu},
$$

se expresa explícitamente de la forma

$$
\begin{aligned}
D_{\mu} \phi & =\partial_{\mu} \phi+i g\left[A_{\mu, \phi}\right] \quad \text { o } \\
D_{\mu} \phi^{a} & =\partial_{\mu} \phi^{a}-g \epsilon_{a b c} A_{\mu}^{b} A_{\nu}^{c} .
\end{aligned}
$$

Luego las ecuaciones de campo correspondientes al lagrangiano, Ec.(16), se expresan como

$$
\begin{aligned}
D_{\nu} F^{a \mu \nu} & =-g \epsilon_{a b c} \phi^{b} D^{\mu} \phi^{c}, \\
D_{\mu} D^{\mu} \phi^{a} & =-\lambda \phi^{a}\left(\phi^{b} \phi^{b}-a^{2}\right) .
\end{aligned}
$$

Como observamos anteriormente, debemos buscar las soluciones estáticas de las ecuaciones de campo, pues éstas corresponden a solitones. Además buscamos soluciones que satisfacen $A_{0}^{a}(x)=0$ para todo $(x, a)$. De manera que las ecuaciones de campo se reducen a las formas

$$
\begin{aligned}
D_{i} F^{a i j} & =g \epsilon_{a b c}\left(D^{j} \phi^{b}\right) \phi^{c} \quad \mathrm{y} \\
D_{i} D^{i} \phi^{a} & =-\lambda\left(\phi^{b} \phi^{b}\right) \phi^{a}+\lambda a^{2} \phi^{a} .
\end{aligned}
$$

Luego, encontramos que la energía para nuestro sistema está dado por

$$
\begin{gathered}
E=\int d^{3} x\left[\frac{1}{4} F_{i j}^{a} F^{a i j}+\right. \\
\left.+\frac{1}{2} D_{i} \phi^{a} D_{i} \phi^{a}+\frac{1}{4} \lambda\left(\phi^{a} \phi^{a}-a^{2}\right)^{2}\right] .
\end{gathered}
$$

Como estamos interesados en las soluciones con energía finita. Se alcanza un mínimo y se anula cuando

$$
F_{i j}^{a}=0, \quad \phi^{a}(x) \phi^{a}(x)=a^{2}, \quad \text { y } \quad D_{i} \phi^{a}=0 .
$$

Estas condiciones definen el vacío de la configuración de Higgs, y la constante $a$ es el valor esperado del vacío del campo escalar.

Establecemos una diferencia entre las posibles soluciones de las ecuaciones de campo que se pueden considerar. El caso trivial es que la orientación de los campos no depende de las coordenadas espaciales y el campo escalar se aproxima asintóticamente a

$$
\phi^{a}=(0,0, a) .
$$

Consideramos, también, otro tipo de soluciones en las cuales la dirección de los campos vectoriales y los campos escalares son función de las coordenadas espaciales. Para construir una solución correspondiente a este caso no trivial consideramos el campo escalar en el límite $r \rightarrow \infty$. Suponemos, que en este límite, el campo escalar está dirigido a lo largo del radiovector del espacio,

$$
\lim _{r \rightarrow \infty} \phi^{a}=\frac{a r^{a}}{r} .
$$

A este tipo de solución, Polyakov [3] denominó hedgehog. Examinando la condición para la derivada covariante, Ec.(24), en $r \rightarrow \infty$ obtenemos

$$
\partial_{n}\left(\frac{r^{a}}{r}\right)-a \epsilon_{a b c} A_{n}^{b} \frac{r^{c}}{r}=0 .
$$

que se transforma en

$$
\begin{aligned}
\partial_{n}\left(\frac{r^{a}}{r}\right) & =\frac{r^{2} \delta_{a n}-r_{a} r_{n}}{r^{3}} \\
& =\frac{1}{r}\left(\delta_{a n} \delta_{c k}-\delta_{a k} \delta_{n c}\right) \frac{r_{c} r_{k}}{r^{2}} \\
& =-\epsilon_{a b c} \epsilon_{b n k} \frac{r_{c} r_{k}}{r^{3}},
\end{aligned}
$$

obteniendo la forma asintótica $(r \rightarrow \infty)$ del potencial de gauge

$$
A_{k}^{a}(r) \longrightarrow \frac{1}{e} \epsilon_{a n k} \frac{r^{n}}{r^{2}} .
$$

Para obtener una solución explícita hacemos uso del ansatz, Ec.(29), de modo que

$$
\begin{aligned}
\phi^{a} & =\frac{r^{a}}{e r^{2}} H(\zeta), \\
A_{n}^{a} & =\epsilon_{a m n} \frac{r^{m}}{e r^{2}}[1-K(\zeta)], \\
A_{0}^{a} & =0,
\end{aligned}
$$

donde $H(\zeta)$ y $K(\zeta)$ son funciones de la variable adimensional $\zeta=e r$. Sustituyendo las ecuaciones (30) en la expresion de la energía, Ec.(23), obtenemos

$$
\begin{aligned}
E & =\frac{4 \pi a}{e} \int_{0}^{\infty} \frac{d \zeta}{\zeta}\left[\zeta^{2}\left(\frac{d K}{d \zeta}\right)^{2}+\frac{1}{2}\left(\zeta \frac{d H}{d \zeta}-H\right)^{2}+\right. \\
& \left.+\frac{1}{2}\left(K^{2}-1\right)^{2}+K^{2} H^{2}+\frac{\lambda}{4 e^{2}}\left(H^{2}-\zeta^{2}\right)\right]
\end{aligned}
$$

Analizando variacionalmente este funcional respecto a las funciones $H$ y $K$ obtenemos que

$$
\begin{aligned}
& \zeta^{2} \frac{d^{2} K}{d \zeta^{2}}=K H^{2}+K\left(K^{2}-1\right) \\
& \zeta^{2} \frac{d^{2} H}{d \zeta^{2}}=2 K^{2} H+\frac{\lambda}{e^{2}} H\left(H^{2}-\zeta^{2}\right),
\end{aligned}
$$


donde estas funciones deben satisfacer las condiciones de frontera

$$
\begin{array}{llll}
K(\zeta) \rightarrow 1, & H(\zeta) & \rightarrow 0 ; & \zeta \rightarrow 0 \\
K(\zeta) \rightarrow 0, & H(\zeta) & \rightarrow \zeta ; & \zeta \rightarrow \infty
\end{array}
$$

que están de acuerdo con las condiciones asintóticas, Ec.(26) y Ec.(29).

De las condiciones de frontera mostradas anteriormente, observamos que para largas distancias

$$
F_{a}^{i j} \longrightarrow \frac{1}{e r^{4}} \epsilon^{i j k} r_{a} r_{k}=\frac{1}{a e r^{3}} \epsilon^{i j k} r^{k} \phi_{a},
$$

lo que implica, en el límite asintótico, que el campo magnético tenga la forma

$$
B \longrightarrow \frac{r_{n} r^{a}}{e r^{4}}
$$

Por comparación con la cuantización del monopolo de Dirac [4], notamos que la carga del monopolo de 't Hootft-Polyakov corresponde al doble de la mínima carga magnética $g=4 \pi / e$. Esto es una consecuencia del hecho de que el grupo U(1) del electromagnetismo se encuentra inmerso en el grupo $\mathrm{SO}(3)$. Esto implica que el monopolo de 't Hooft-Polyakov describe un objeto de dimensiones finitas.

\section{Instantones en Teoría de Campos}

El término instantón se refiere a una solución localizada de energía finita para la ecuación de campo en el espacio euclideano. Esto implica transformar las ecuaciones de campo del espacio de Minkowski al de Euclides.

Para visualizar el proceso de transformación usamos como ejemplo el modelo de doble pozo de potencial, el lagrangiano considerando este potencial es descrito como

$$
\mathcal{L}=\frac{1}{2}\left(\partial_{t} \phi\right)^{2}-\frac{1}{4} \lambda\left(\phi^{a} \phi^{a}-a^{2}\right)^{2}
$$

La acción en el espacio euclideano de esta configuración es obtenida bajo una rotación de Wick dada por

$$
\tau \longrightarrow-i t
$$

de modo que la acción se expresa como

$$
S_{E}=\int d^{d} x d \tau\left[\frac{1}{2}\left(\partial_{\tau} \phi\right)^{2}+\frac{1}{4} \lambda\left(\phi^{a} \phi^{a}-a^{2}\right)^{2}\right] .
$$

Por el método de los extremales, obtenemos que

$$
\partial_{\tau}^{2} \phi=\frac{d V}{d \phi}=\lambda\left(\phi^{3}-a^{2} \phi\right)
$$

resolviendo la ecuación

$$
\frac{d \phi}{d \tau}=(2 V)^{\frac{1}{2}}
$$

obtenemos que

$$
\phi(\tau)= \pm a \tanh \left[a \sqrt{\frac{\lambda}{2}}\left(\tau-\tau_{0}\right)\right] .
$$

De esta forma obtenemos los instantones y los antiinstantones para el doble pozo de potencial. Por otro lado, notamos que estas soluciones para la acción finita nos remiten a las soluciones estáticas para una configuración de energía finita en el espacio de Minkowski, kink y anti-kink.

Luego, generalizamos el proceso para un potencial $V$ en el lagrangiano, determinamos la acción para una configuración $(d, 1)$ de modo que se expresa como

$$
S=\int d^{d} x d t\left[\frac{1}{2} \partial_{t} \phi \partial_{t} \phi-\frac{1}{2} \partial \phi \partial \phi-V(\phi)\right],
$$

la cual requiere ser finita y bajo una transformación de Wick obtenemos la acción en el espacio euclideano

$$
S_{E}=\int d^{d} x d \tau\left[\frac{1}{2} \partial_{t} \phi \partial_{t} \phi+\frac{1}{2} \partial \phi \partial \phi+V(\phi)\right] .
$$

Cabe notar que para el caso de los instantones se requiere que $S_{E}$ sea finita. Mientras que, para el caso de los solitones se requiere que la energía sea finita

$$
E=\int d^{D} x\left[\frac{1}{2} \partial_{t} \phi \partial_{t} \phi+\frac{1}{2} \partial \phi \partial \phi+V(\phi)\right] .
$$

Por tanto, se determina la correspondencia entre una solución instantónica y una solución solitónca estática, obteniendo además la condición de equivalencia, $d+1=D$.

\section{Instantones en Teorías de Yang-Mills}

La acción en el espacio euclideano se describe como

$$
S=-\frac{1}{2 g^{2}} \int d^{4} x \operatorname{Tr}\left(F_{\mu \nu} F^{\mu \nu}\right),
$$

y la ecuación para un campo puro de Yang-Mills se expresa como

$$
\partial_{\nu} F_{\mu \nu}+i\left[F_{\mu \nu}, A_{\nu}\right]=0 .
$$

Los instatones son soluciones clásicas en el espacio euclideano con energía finita del campo de Yang-Mills. El interés por este tipo de configuración tiene como objetivo hacer una aproximación semiclásica [5], esto implica que $F_{\mu \nu}$ debe disminuir más rápidamente que $1 /\left|x^{2}\right|$ cuando $|x| \rightarrow \infty$. Ahora partiendo de la identidad

$$
F_{\mu \nu} F^{\mu \nu}=\widetilde{F}_{\mu \nu} \widetilde{F}^{\mu \nu}
$$

la acción se expresa como

$$
S=-\frac{1}{4 g^{2}} \int d^{4} x \operatorname{Tr}\left[F_{\mu \nu} F^{\mu \nu}+\widetilde{F}_{\mu \nu} \widetilde{F}^{\mu \nu}\right] .
$$


La condición de anti-autodualidad [6] expresa que

$$
\begin{gathered}
S=-\frac{1}{2 g^{2}} \int d^{4} x \operatorname{Tr}(F \pm \widetilde{F})^{2} \pm \frac{1}{2 g^{2}} \int d^{4} \operatorname{Tr} F_{\mu \nu} \widetilde{F}^{\mu \nu} \\
\text { y } \quad S \geq \pm \frac{1}{2 g^{2}} \int d^{4} x \operatorname{Tr} F_{\mu \nu} \widetilde{F}^{\mu \nu}
\end{gathered}
$$

la igualdad satisface si el tensor de campo es antiautodual.

Para encontrar las soluciones de los instantones en el espacio euclideano usamos la condición de autodualidad

$$
F^{\mu \nu}= \pm \widetilde{F}^{\mu \nu} .
$$

La elección de la condición de autodualidad o antiautodualidad está relacionado con el número de enrrollamiento de la solución. El número de enrrollamiento es una cantidad que se conserva en el sistema y no es una cantidad dinámica, es decir, no esta relacionada con el teorema de Noether, sino se encuentra relacionada con la topología de las soluciones [7], [5].

El siguiente Ansatz para el campo de gauge dado por

$$
A_{\mu}(x)=\alpha \sigma_{\mu \nu} \partial_{\nu} \ln \phi\left(x^{2}\right),
$$

donde $\alpha$ es una constante real y $\sigma_{\mu \nu}$ la representación matricial $2 \times 2$ de los generadores de Lorentz en el espacio euclidiano es introducido en la definición del tensor de campo y utilizando las propiedades de anticonmutación de los generadores de Lorentz [8] obtenemos que

$$
F_{\mu \nu}=\partial_{\mu} A_{\nu}-\partial_{\nu} A_{\mu}+\left[A_{\mu}, A_{\nu}\right],
$$

desarrollando detalladamente, tenemos

$$
\begin{gathered}
F_{\mu \nu}=\left[\alpha \sigma_{\nu \mu} g_{\nu} \partial_{\mu} \ln \phi-(\nu \leftrightarrow \mu)\right]+ \\
+\left[2 \alpha^{2} \sigma_{\nu \sigma} \partial_{\nu} \ln \phi \partial_{\sigma} \ln \phi-(\nu \leftrightarrow \sigma)\right]- \\
-2 \alpha^{2} \sigma_{\nu \mu}(\partial \ln \phi)^{2},
\end{gathered}
$$

y para el tensor dual de campo

$$
\begin{gathered}
\widetilde{F}_{\mu \nu}=\frac{1}{2} \epsilon_{\nu \mu \rho \sigma} F_{\rho \sigma}=\sigma\left(\alpha \partial_{\sigma} \partial_{\nu} \ln \phi-\right. \\
\left.-2 \alpha^{2} \partial_{\rho} \ln \phi \partial_{\mu} \ln \phi\right)-(\nu \leftrightarrow \mu) .
\end{gathered}
$$

Aplicando la condición de auto-dualidad

$$
\alpha \partial^{2} \ln \phi+2 \alpha(\partial \ln \phi)^{2}=0,
$$

se establece que $\alpha=\frac{1}{2}$, de modo que

$$
\begin{aligned}
\partial^{2} \ln \phi+(\partial \ln \phi)^{2} & =0 \\
y \frac{\partial^{2} \phi}{\phi} & =0 .
\end{aligned}
$$

Cuando $\phi$ es no-singular, la Ec.(56) se reduce a $\partial^{2} \phi=0$, de modo que $\phi=$ constante, conduciendo a que $A_{\mu}=0$. Pero cuando $\phi(x)$ es singular obtenemos soluciones no-singulares para el campo de gauge dado como

$$
\phi=1+\sum_{i=1}^{k} \frac{\rho^{2}}{\left(x-a_{i}\right)^{2}},
$$

y obtenemos

$$
A_{\mu}=-\sigma_{\mu \nu} \frac{\rho^{2}(x-a)_{\nu}}{(x-a)^{2}\left((x-a)^{2}+\rho^{2}\right)},
$$

observando que $A_{\mu}$ es singular en el punto $x=a$ y para puntos en la vecindad $x \approx a$ tenemos que

$$
A_{\nu}(x)=-\sigma_{\mu \nu} \frac{(x-a)_{\nu}}{(x-a)^{2}},
$$

que es un campo de gauge puro que se describe también como

$$
U^{-1} \partial_{\mu} U=-\sigma_{\mu \nu} \frac{(x-a)_{\nu}}{(x-a)^{2}}
$$

reescribimos

$$
\begin{aligned}
A_{\mu}(x) & =A_{\mu}(x) \frac{\rho^{2}}{(x-a)^{2}+\rho^{2}} \\
& =\frac{\rho^{2}}{(x-a)^{2}+\rho^{2}} U^{-1} \partial_{\mu} U,
\end{aligned}
$$

que al usar la transformada de gauge inversa

$$
A^{\prime}=U \partial_{\mu} U^{-1}+U^{-1} \partial_{\mu} U
$$

obtenemos

$$
A^{\prime}=-\sigma_{\mu \nu} \frac{(x-a)_{\nu}}{(x-a)^{2}+\rho^{2}} .
$$

Esta es la solución tipo instantón. Esta solución no presenta singularidades para algún valor de $x$ o para algún $\rho=0$. Nótase que solo hemos obtenido soluciones para la condición de autodualidad. De manera análoga obtenemos las soluciones para la condición de anti-autodualidad dada por

$$
F_{\mu \nu}+\widetilde{F}_{\mu \nu}=0
$$

que reemplazamos en el ansatz, Ec.(51), así como la condición $\sigma_{\mu \nu}$ por $\bar{\sigma}_{\mu \nu}$ obteniendo la solución

$$
A^{\prime}=-\bar{\sigma}_{\mu \nu} \frac{(x-a)_{\nu}}{(x-a)^{2}+\rho^{2}} .
$$

Comparando con la definición tenemos que

$$
\begin{aligned}
A=A_{\mu}^{a} \frac{\tau_{a}}{2 i} & =-\bar{\sigma}_{\mu \nu} \frac{(x-a)_{\nu}}{(x-a)^{2}+\rho^{2}}, \\
A_{\mu}^{a}(x) & =2 \eta_{\mu \nu}^{a} \frac{(x-a)_{\nu}}{(x-a)^{2}+\rho^{2}}
\end{aligned}
$$

donde $\eta=-i \tau_{a} \bar{\sigma}_{\mu \nu}$ son los símbolos de 't Hooft [7] 


\section{Conclusiones}

A diferencia del monopolo de Dirac, las soluciones monopolares en teorías de Yang-Mills son obtenidas sin la introducción de fuentes de carga magnética. Estas soluciones son estables y deberían ser observadas en la naturaleza como objetos materiales. Sin embargo, los monopolos en el $\mathrm{SU}(2)$ posiblemente poseen masas de varios $\mathrm{GeV}$ que hace imposible su medición experimental. No obstante, sus propiedades matemáticas pueden ser estudiadas independientemente.

Uno de los resultados más importantes de las teo- rías de Yang-Mills es la obtención de soluciones instantónicas para la ecuación de movimiento. Es importante notar que no hemos demostrado que todas las soluciones con acción finita para la Ec.(45) son de tipo antiautodual.

La existencia de soluciones no autoduales con acción finita ha sido obtenida para diferentes números de enrrollamiento $[9,10]$. Esto implica que el vacío de Yang-Mills consiste de diferentes sectores con carga topológica relacionados entre sí, por tunelaje instantónico $[7,11]$.

\section{Referencias}

[1] C.N. Yang y R.L. Mills, Conservation of Isotopic Spin and Isotopic Gauge Invariance, Phys. Rev. 96, 191 (1954).

[2] G. 't Hooft, Magnetic monopoles in unified gauge theories, Nuclear Physics B 79, 276 (1974).

[3] A.M. Polyakov, Particle spectrum in quantum field theory, JETP Lett. 20, 194 (1974).

[4] P.A.M. Dirac, Quantised Singularities in the Electromagnetic Field, Proc. Roy. Soc. A 133, 60 (1931).

[5] S. Coleman, Aspects of Symmetry, Cambridge University Press, Cambridge (1985).

[6] A.A. Belavin, A.M. Polyakov, A.S. Schwartz y Yu. S. Tyupkin, Pseudoparticle solutions of the Yang-Mills equations, Phys. Lett. B 59, 85, (1975).

[7] R. Rajaraman, Solitons and Instantons: An Introduction to Solitons and Instantons in Quantum Field Theory, North-Holland, Amsterdam (1989).

[8] S. Vandores y P. Van Nieuwenhuizen, Lectures on instantons, arXiv:0802.1862v1 [hep-th], (2008).

[9] L. Sadun y J. Segert, Non-self-dual Yang-Mills connections with non-zero Chern number, Bull. Amer. Math. Soc. 24, 163 (1991).

[10] L. Sadun y J. Segert, Stationary point of the Yang-Mills action, Commun. Pure Appl. Math. 45, 461 (1992).

[11] A.I. Vaŭnshtĕn, V.I. Zakharov, V. A Novikov y M.A. Shifman, $A B C$ of Instantons, Sov. Phys. Usp. 25, 195 (1982). 